\title{
1-MCP Improved Diverse Sensorial, Biochemical and Physical Apple Traits during Cold Storage Based on Cultivar
}

\author{
B. Pashazadeh ${ }^{1}$, H. Hajnajari ${ }^{2}$, F. Shavakhi ${ }^{3}$ \\ ${ }^{1}$ Post Harvest Group, Sabzevar Branch, Islamic Azad University, Sabzevar, Iran \\ ${ }^{2}$ Temperate Fruit Research Center, Horticulture Science Research Institute, Agricultural Research, Education and Extension \\ Organization (AREEO), Karaj, Iran \\ ${ }^{3}$ Agricultural Engineering Research Institute, Agricultural Research Education and Extension Organization (AREEO), Karaj, Iran \\ Email: `hassanhajnajari@yahoo.com, h.hajnajari@areeo.ac.ir
}

How to cite this paper: Pashazadeh, B., Hajnajari, H. and Shavakhi, F. (2017) 1-MCP Improved Diverse Sensorial, Biochemical and Physical Apple Traits during Cold Storage Based on Cultivar. Agricultural Sciences, 8, 77-96.

http://dx.doi.org/10.4236/as.2017.81007

Received: November 24, 2016

Accepted: January 20, 2017

Published: January 23, 2017

Copyright $\odot 2017$ by authors and Scientific Research Publishing Inc. This work is licensed under the Creative Commons Attribution International License (CC BY 4.0).

http://creativecommons.org/licenses/by/4.0/

\begin{abstract}
Apple cultivars' storability could be strengthened through known techniques of cold storage, advanced industries of control atmosphere or other routine usages like fruit pre-cooling followed by immersion in $\mathrm{CA}^{++}$solution, depending on genetic potential of the plant material. At the end of pomology and cold storage assessments of 93 apple cultivars, two high yielding native "Dirras-e Mashhad" and "Wealthy" were treated by single and combined 1-MCP and ethylene nano-absorbent (ENA) to assess eventual improvements in cold storage span, fruit eating quality and role of cultivar. For this purpose, directs effects and interactions between all sources of variance were studied. Ethylene emission and $\mathrm{CO}_{2}$ amounts were measured. The results showed that reduced weight loss and flesh firmness, were registered in the treated samples of both cultivars compared with the control. Using of 1-MCP affected significantly ( $\mathrm{p}<0.05)$ TSS (Total Soluble Solids), $\mathrm{pH}$ and TA (Titratable Acidity). 1-MCP inhibited rise of the fruit $\mathrm{pH}$, decreasing also the level of TA during storage, while using of ethylene nano-absorbent showed no significant effect ( $p>0.05)$ on these characteristics. The native apple "Dirras-e Mashhad" showed higher $\mathrm{pH}$ value than "Wealthy" under the combined treatment (ENA+1-MCP). Genetic source revealed significant difference through lower "Wealthy" storability. Ethylene emission increased to $53.64 \mathrm{ppm} / \mathrm{Kg} \cdot \mathrm{hr}$ after $5^{\text {th }}$ month, which resulted in significant difference at $\mathrm{p}>0.01$ level related to the initial value of $3.26 \mathrm{ppm} / \mathrm{Kg} \cdot \mathrm{hr}$. The combined treatment (ENA+1-MCP) affected significantly $(\mathrm{p}<0.05)$ most of the fruit characteristics in both of the cultivars. The treatments demonstrated different results by cultivar.
\end{abstract}




\section{Keywords}

1-MCP, Ethylene Nano Absorbent, Flesh Softening, Storage Life

\section{Introduction}

Apple (Malusxdomestica Borkh.) is one of the most cultivated fruit trees worldwide, with production of $69,511,975$ tons for which Iran has been variously ranked amid third to eighth global producer with annual production of approximately 2 to 1,662,430 tons in the high competitive market (FAO, 2012) [1]. The apple industries have encountered a period of intense competition, described as being in a state of hypercompetition (Harker et al. 2003) [2]. Waste control in such delicate market reflects as a priority particularly for a climacteric crop. Apple respiration rate is influenced by factors including temperature, storage conditions expressed by oxygen, carbon dioxide and ethylene concentrations. Ethylene regulates important biological activities of fruit growth, maturation affecting aging process and senescence leading to fruit fall through formation of abscission layer (Rahemi, 2004) [3]. 1-MCP (1-methyl cyclopropene), despite the similar chemical layout related to ethylene, demonstrates anti-ethylenic properties. It is known as relatively new worthy compound due to its non-toxic influence on biological systems, inexpensive and high efficiency related to other ethylene reductive methods. It is retained to increase storage life and preserve fruit quality through decreasing the rate of ethylene production and consequent decelerating respiration rhythm leading to delay in ripening time. Delayed aging is accompanied with less flesh softening and not as much of color change. 1-MCP, $\mathrm{C} 4 \mathrm{H} 6$ is able to maintain the quality and reduce the incidence of physiological disorders under limited temperature and normal pressure (Pelayo et al. 2005) [4]. Respiration occurs more in actively growing plant tissues than mature parts with a minimum in dormant organs, assuming that its rate will be duplicated in each increase of temperature of $10^{\circ} \mathrm{C}$, only if $\mathrm{O}_{2}$ shortage and/or accumulation of $\mathrm{CO}_{2}$ is inhibited. Ethylene synthesis, as a gaseous senescence hormone, is impeded also by aminoethoxyvinylglycine (AVG), a highly efficient chemical in decreasing respiration rate and carbon dissimilation (Tromp et al. 2005) [5]. Pre-harvest spray of daminozide is also recommended because of significant improvements in the quality of apples stored in controlled atmospheres (Sharples \& Johnson, 1986) [6]. Other ethylene inhibitors such as potassium permanganate and ozone are considered as strong oxidizers (Jalili Marandi, 2008) [7]; the non-volatile compounds can be easily separated from the product. In a long-term trial, the fruit samples of six most diffused apple cultivars in Europe as "Braeburn", "Cripps Pink", "Gala", "Fuji", "Golden Delicious", "Red Delicious" were harvested at three successive stages of fruit growth as beginning, mid and physiological maturity and stored in controlled atmosphere (CA) for 5 to 7.5 months under ultra low oxygen and/or CA storage conditions with and without prior exposure to 1-MCP SmartFresh ${ }^{\mathrm{TM}}$. "Golden Delicious" 
responded well in fruit firmness increase when stored in the more advanced storage technique using 1-MCP or DCA-CF, related to low oxygen. Both techniques could also increase fruit firmness of "Braeburn", but DCA-CF storage proved to be more effective on the latter cultivar than the 1-MCP treatment (Zanella \& Rossi, 2015) [8]. 1-MCP pre-storage treatments entailed positive changes in sensory properties of "Granny Smith" apples during cold storage along with subsequent keeping of fruit at room temperature. At the end of cold storage, 0 $1^{\circ} \mathrm{C}, 90$ - 95 relative humidity (RH), apples were stored in normal atmosphere 1 , 8 and 15 and evaluated after 3, 6 and 9 months (Tomic et al. 2016) [9]. Ethylene removal is feasible by wide exposition to potassium permanganate. For this purpose, saturated solution of potassium permanganate should be laid on safe bed minerals like inflated mica, selenite, silicagel, alumina plates, perlite and expanding glasses. The plates containing potassium permanganate set in filtered sachets absorb ethylene molecules when air filtered (Zomorrodi, 2005) [10]. The effects of 1-MCP and ENA granules, singularly and in multiple combinations, on storage life and preserving the qualitative characteristics of two apple cultivars were investigated.

\section{Materials \& Methods}

\subsection{Plant Material}

The fruit samples of two apple cultivars, Iranian native "Dirras-e Mashhad" and imported "Wealthy", were collected from the 20-year-old vase form trees on seed rootstocks grown in Karaj-Iran climatic conditions. The National collection of commercial apple cultivars is established in Horticultural Research Station of KamalShahr under Temperate Fruit Research Center. In late September of 2010, sampling was performed based on precise harvest time by assessment of the starch index $(2-2.5)$. At picking, the fruits were gently twisted without exerting any pressure by finger tips and slowly placed in appropriate boxes under the trees shadow. The harvested fruits passed 24 hours of pre-cooling and then were stored at $0.5^{\circ} \mathrm{C}$ and $90 \%$ of relative humidity.

\section{2. "Dirras-e Mashhad" Traits}

Origin: Mashahd, Iran, late ripening, days from bloom to pick: 161, the mean of 10 fruits: globose-oblong globose, diameter $66.1 \mathrm{~mm}$, length/diameter ratio 0.83 , russet susceptibility class 2, background: green, over-color: red, stalk length: 10 $\mathrm{mm}$, peel thickness: medium-thick, seed size: medium, total seed/fruit and carpel: 7.5 and 5 .

\subsection{Wealthy Traits}

Origin: Minnesota, USA, late ripening, days from bloom to pick: 156, the mean of 10 fruits: globose, diameter $62.5 \mathrm{~mm}$, length/diameter ratio 0.79 , russet susceptibility class 3, background: green, over-color: red, stalk length: $9 \mathrm{~mm}$, peel medium-thick, seed size: big, mean total seed/fruit and carpel: 4.5 and 4.5 . 


\section{Treatments}

The experiment was achieved by studying the influence of three different chemical treatments related to control. In the first treatment, the samples were sprayed by $1 \mathrm{ppm}$ of $1-\mathrm{MCP}$, made by LupoFresh Inc., imperviously for 8 hours at room temperature in gas exchange ambient. The treated samples by 1-MCP after 1 hour aeration were placed inside the boxes together with sachets containing 5-gram small granules of ENA manufactured by BioConservation Company. The second treatment was defined by single use of 1-MCP treated apples without ENA sachets and the third one by sole ENA sachets. The control samples were stored with no chemical treatment in normal conditions. Special shoulders containing apples, three repetitions for each treatment, placed inside the boxes were transferred to cold room at $0.5^{\circ} \mathrm{C}$ and $90 \%$ R.H. In each experiment, 30 randomly selected fruits per cultivar were ordered in three distinct replicates of 10 fruit each. During 5 months of cold storage, qualitative and quantitative characteristics were evaluated. The effects of three different treatments were compared to control in each cultivar.

\subsection{Weight Loss}

Fruit weight was recorded by a digital scale with a precision of 0.1 gram, model B502 College. To measure the weight loss, the treated and control fruits of each cultivar weighed in separated boxes and compared to the initial weight. The measurements were computed in monthly temporal intervals for weight loss unto fifth month of cold storage.

\subsection{Flesh Firmness}

Flesh firmness was assessed by the Texture Analyzer, Hounsfield-H5KS model made in England. Thin layer of fruit peel sole per $1 \mathrm{~cm}^{2}$ was removed in 3 points by a sharp blade where a cylindrical plunger, $6.4 \mathrm{~mm}$ diameter, was pushed into apple flesh tissue, measured in Newton, over a constant distance with a speed of $20 \mathrm{~mm}$ per min (Finny \& Massie, 1975) [11].

\section{Instrumental Measurements of Biochemical Traits TSS, TA and pH}

Once removed ovary tissues, the juice was prepared by squeezing longitudinal slices of 1.5 fruit diameter of 10 fruits from each replication in juicer. The apple juice was passed through the filter paper and collected then into a flask Pre(Aymard et al. 2005) [12], TSS, pH and TA values of the two cultivars juice were determined for the defined treatments based on experimental design. TA was measured using caustic soda, 0.1 through the normal formula calculated on the basis of predominant malic acid in apple flesh, equivalent to 67 grams (Watkins, 2003 [13]; Hosseini, 1990 [14]). After calibrating pH meter with buffer solutions, $\mathrm{pH}$ of $20 \mathrm{mg} / \mathrm{l}$ of pure fruit juice was measured by digital $\mathrm{pH}$ meter. Few drops of filtered fruit juice put on the ESR prism surface was read in sort of manual ATAGO refractometer model 20181MC ranges (Brix $0 \sim 32 \%$ ). The measure- 
ments were computed in monthly temporal intervals for biochemical traits unto fifth month of cold storage and the data were registered.

\subsection{Sensory Evaluation}

Taste-panel assessments founded on purely biological means could not be replaced by instrumental tests. In order to appraise sensorial traits, sound clean fruit samples of each cultivar were distributed to the 10 well-trained panelists as fixed members of the group, before lunch. Appraisements carried out by scoring flavor, aroma, juiciness, taste (sweetness, sourness, acidulous) and general acceptability. Acceptability index represents overall sensorial perception of the panelists, in predefined assessment sheets rated between 0 to 100 where index value 0 indicates dissatisfaction and 100 high satisfactions included all intermediate scales.

\subsection{Data Analysis}

To evaluate the eventual increase of storage life and maintaining the quality characteristics of two apple fruit cultivars by use of 1-MCP, a factorial experiment with a treatment of 1-MCP and ENA on two apple cultivars wealthy and Dirras-e Mashhad was applied within completely randomized design. Analysis of variance (ANOVA) was performed using SAS statistical software. Mean comparisons were performed using Duncan's Multiple Range test, all triplicates.

\subsection{Ethylene Measurements}

The biosynthesized ethylene amount per volume was achieved by ethylenesensitive sensor (Bioconservation, UK). Measured the weight (g) and volume $\left(\mathrm{cm}^{3}\right)$ of the fruits, three samples were placed into a 1 liter glass vase and $16 \mathrm{~h}$ later the values of the gaseous ethylene were registered in ppm.

\section{6. $\mathrm{CO}_{2}$ Measurements}

The $\mathrm{CO}_{2}$ produced was quantified using equipment Testo Model 435 made in Germany. The analysis was performed using 3 apples weighed by digital balance College B502 then placed in sealing chamber. The emitted $\mathrm{CO}_{2}$ was estimated in ppm during $30 \mathrm{~min}$. scattered per $1 \mathrm{~min}$. intervals. The data were collected for computations.

\section{Results}

The analysis of variance showed that biochemical characteristics and physical traits of the two cultivars were affected differently by use of sole 1-MCP or ENA and the combined form 1-MCP + ENA during storage (Table 1).

\subsection{Fruit Weight Loss}

The mean comparisons of weight loss percent between cultivars by use of 1$\mathrm{MCP}, \mathrm{ENA}$ and also combined treatment (1-MCPe + ENA) showed the highest 
Table 1. Analysis of variance for the effects of different treatments on qualitative apple characteristics.

\begin{tabular}{|c|c|c|c|c|c|c|c|}
\hline \multirow{2}{*}{ Source of variance } & \multirow{2}{*}{ DF } & \multicolumn{3}{|c|}{ Square means } & \multirow[b]{2}{*}{ Fmax } & \multirow[b]{2}{*}{ DF } & \multirow[b]{2}{*}{ Weight loss } \\
\hline & & $\mathrm{pH}$ & TA & TSS & & & \\
\hline Storage time & 5 & $0.31^{\star *}$ & $0.99^{\star *}$ & $28.53^{* \star}$ & $1790.2^{\star \star}$ & 4 & $7.54^{\star \star}$ \\
\hline Cultivar & 1 & $2.96^{* *}$ & $15.79^{* *}$ & $246.12^{\star \star}$ & $7929.1^{\star *}$ & 1 & ${ }^{\star} 0.73$ \\
\hline ENA & 1 & $0.11 \mathrm{~ns}$ & $0.97^{* *}$ & $19.17^{* *}$ & $14.19 \mathrm{~ns}$ & 1 & $0.0035 \mathrm{~ns}$ \\
\hline 1-MCP & 1 & $0.12 \mathrm{~ns}$ & $0.51 \mathrm{~ns}$ & $0.57 \mathrm{~ns}$ & $427.01^{\star *}$ & 1 & $1.02^{* *}$ \\
\hline Cultivar $\times$ Time & 5 & $0.14^{* *}$ & $0.51^{\star *}$ & $12.59^{* *}$ & $324.15^{* *}$ & 4 & $1.26^{* *}$ \\
\hline ENA $\times$ Time & 4 & $0.005 \mathrm{~ns}$ & $0.09 \mathrm{~ns}$ & $0.0001 \mathrm{~ns}$ & $54.02^{* \star}$ & 4 & $0.56^{* *}$ \\
\hline $1-\mathrm{MCP} \times$ Time & 4 & $0.13^{\star *}$ & $0.31^{\star *}$ & $3.02^{* *}$ & $0.86 \mathrm{~ns}$ & 4 & $0.86^{\star *}$ \\
\hline ENA $\times$ Cultivar & 1 & $0.11 \mathrm{~ns}$ & $0.0004 \mathrm{~ns}$ & $32.87^{\star *}$ & $18.85 \mathrm{~ns}$ & 1 & $0.029 \mathrm{~ns}$ \\
\hline 1-MCP $\times$ Cultivar & 1 & $0.11 \mathrm{~ns}$ & $0.024 \mathrm{~ns}$ & $0.257 \mathrm{~ns}$ & $12.76 \mathrm{~ns}$ & 1 & $0.051 \mathrm{~ns}$ \\
\hline $\mathrm{ENA} \times 1-\mathrm{MCP}$ & 1 & $0.001 \mathrm{~ns}$ & $0.051 \mathrm{~ns}$ & $6.54^{\star *}$ & $0.0067 \mathrm{~ns}$ & 1 & $0.0067 \mathrm{~ns}$ \\
\hline ENA $\times$ Cultivar $\times$ Time & 4 & $0.059^{* *}$ & $0.13^{* *}$ & $0.004 \mathrm{~ns}$ & $29.03 \mathrm{~ns}$ & 4 & $1.3^{* *}$ \\
\hline $1-\mathrm{MCP} \times$ cultivar $\times$ Time & 4 & $0.068^{* *}$ & ${ }^{*} 0.81$ & $3.39^{* *}$ & $50.93^{* *}$ & & $1^{\star *}$ \\
\hline ENA $\times 1-\mathrm{MCP} \times$ Time & 4 & $0.026^{* *}$ & $0.51^{* *}$ & $6.12^{* *}$ & $31.97 \mathrm{~ns}$ & 4 & $0.59^{* *}$ \\
\hline ENA $\times 1-\mathrm{MCP} \times$ Cultivar & 1 & $0.088^{* *}$ & $0.18^{* *}$ & $2.34^{* *}$ & $6.17 \mathrm{~ns}$ & 4 & $0.139 \mathrm{~ns}$ \\
\hline Error & 88 & 0.0038 & 0.0069 & 0.07 & 14.35 & 1 & 0.112 \\
\hline CV \% & & 1.47 & 2.38 & 1.8 & 25.33 & 84 & 15.72 \\
\hline
\end{tabular}

${ }^{*},{ }^{* *}$, ns: significant at 1, 5 percent and none-significant respectively. DF: Degree of freedom.

decline, significant at $\mathrm{p}<0.05$ level, in "Wealthy" at 5th month of storage related to "Dirras-e Mashhad" due to non treated samples by 1-MCP and or ENA, while no significant difference was noted during the first 3 months (Tables 2-4). The 1-MCP efficacy was evaluated on quality of "Cortland" and "Empire" apples after storage. The treated samples for 3 hours at any of the temperatures reduced significantly the incidence of superficial scald sole in "Cortland". The final results indicated very high potential of 1-MCP for keep flesh quality during storage; nevertheless its efficacy could be influenced by temperature and duration as well as by apple cultivar (De Ella et al. 2002) [15]. Weight loss comparisons caused by single treatments of 1-MCP and ENA in both of the cultivars during storage time affirmed highest loss in "Wealthy" during the fifth month by ENA presence and lack of 1-MCP, while in the first four months the two cultivars had no significant difference (Table 2, Table 3). The combined treatments of the compounds showed no significant differences during storage (Table 5).

\subsection{Effect of Cultivar and ENA on Flesh Firmness}

Genetic variability had imminent role in flesh firmness trait so that though the mean comparisons showed decreasing trend for both of the cultivars during storage but "Dirras-e Mashhad" presented lower values related to "Wealthy" at the end of all five stepwise storage months. Also the difference levels varied clearly 
Table 2. Mean comparisons of ENA and cultivar interaction during storage time on the two apple cultivars qualitative characteristics.

\begin{tabular}{|c|c|c|c|c|c|c|c|}
\hline Storage time & Cultivar & ENA & $\mathrm{pH}$ & Acidity & TSS Brix & Weight Loss & Fmax \\
\hline \multirow[t]{3}{*}{ Starting } & Dirras-e Mashhad & - & $4.56 \mathrm{a}$ & $2.96 \mathrm{~m}$ & 9.61 & & $19.07 \mathrm{c}$ \\
\hline & Wealthy & - & $4.03 \mathrm{fg}$ & $4.7 \mathrm{a}$ & $11 \mathrm{k}$ & & $19.05 \mathrm{c}$ \\
\hline & Dirras-e Mashhad & - & $4.28 \mathrm{~d}$ & $3.75 \mathrm{ef}$ & $15.5 \mathrm{~d}$ & $0.30 \mathrm{abc}$ & $25.08 \mathrm{a}$ \\
\hline \multirow[t]{4}{*}{ 1st month } & & + & $4.38 \mathrm{bc}$ & $3.48 \mathrm{hi}$ & $16 \mathrm{c}$ & $0.11 b c$ & $27.08 \mathrm{a}$ \\
\hline & Wealthy & - & $4.42 \mathrm{~b}$ & $4 \mathrm{bc}$ & $13.5 \mathrm{~h}$ & $0.43 a b c$ & $13.07 \mathrm{de}$ \\
\hline & & + & $4.17 \mathrm{e}$ & $4.1 \mathrm{~b}$ & $13 \mathrm{i}$ & $0.65 a b c$ & $13.08 \mathrm{de}$ \\
\hline & Dirras-e Mashhad & - & $4.32 \mathrm{~cd}$ & $2.98 \mathrm{~lm}$ & $15.5 \mathrm{~d}$ & $0.5 \mathrm{abc}$ & $15.9 \mathrm{~d}$ \\
\hline \multirow[t]{4}{*}{ 2nd month } & & + & $4.38 \mathrm{bc}$ & $3.26 \mathrm{j}$ & $17.6 \mathrm{a}$ & $0.46 \mathrm{bc}$ & $16.5 \mathrm{~d}$ \\
\hline & Wealthy & - & $4.09 \mathrm{f}$ & $3.43 \mathrm{i}$ & $12.6 \mathrm{j}$ & $0.05 \mathrm{bc}$ & $10.1 \mathrm{fg}$ \\
\hline & & + & $4.04 \mathrm{f}$ & $3.73 \mathrm{fe}$ & $12.5 \mathrm{j}$ & $0.90 \mathrm{ab}$ & $10.5 \mathrm{fg}$ \\
\hline & Dirras-e Mashhad & - & $4.45 \mathrm{~b}$ & $2.98 \mathrm{~lm}$ & $16.4 \mathrm{~b}$ & $0.25 a b c$ & $7.3 \mathrm{~h}$ \\
\hline \multirow[t]{4}{*}{ 3rd month } & & + & $4.31 \mathrm{~cd}$ & $3.1 \mathrm{kl}$ & $17.3 \mathrm{a}$ & $0.42 a b c$ & $10.5 \mathrm{fg}$ \\
\hline & Wealthy & - & $4.06 \mathrm{f}$ & $3.65 f g$ & $12.3 \mathrm{j}$ & $0.29 a b c$ & $4.1 \mathrm{i}$ \\
\hline & & + & $3.96 \mathrm{~g}$ & $4.03 \mathrm{bc}$ & $12.3 \mathrm{j}$ & $0.13 b c$ & $3.8 \mathrm{i}$ \\
\hline & Dirras-e Mashhad & - & $4.42 \mathrm{~b}$ & $3.25 \mathrm{j}$ & $16 \mathrm{c}$ & $0.29 \mathrm{abc}$ & $28.2 \mathrm{a}$ \\
\hline \multirow[t]{4}{*}{ 4th month } & & + & $4.38 \mathrm{bc}$ & $2.95 \mathrm{~m}$ & $16.6 \mathrm{~b}$ & $0.39 a b c$ & $23.7 \mathrm{~b}$ \\
\hline & Wealthy & - & $4.10 \mathrm{ef}$ & $3.93 \mathrm{~cd}$ & $14.6 \mathrm{f}$ & $0.41 \mathrm{abc}$ & $12.5 \mathrm{ef}$ \\
\hline & & + & $4.04 \mathrm{f}$ & $3.8 \mathrm{e}$ & $14 \mathrm{~g}$ & $0.16 \mathrm{bc}$ & $10.9 \mathrm{fg}$ \\
\hline & Dirras-e Mashhad & - & $4.26 \mathrm{~d}$ & $2.6 \mathrm{n}$ & $15.4 \mathrm{~d}$ & $0.40 \mathrm{abc}$ & $20.1 \mathrm{c}$ \\
\hline \multirow[t]{3}{*}{ 5th month } & & + & $4.17 \mathrm{e}$ & $3.16 \mathrm{jk}$ & $17.5 \mathrm{a}$ & $0.57 \mathrm{abc}$ & $19.1 \mathrm{c}$ \\
\hline & Wealthy & - & $3.72 \mathrm{~h}$ & $3.58 \mathrm{gh}$ & $15 \mathrm{e}$ & $0.47 \mathrm{abc}$ & $8.8 \mathrm{gh}$ \\
\hline & & + & $3.77 \mathrm{~h}$ & $3.83 \mathrm{ed}$ & $14 \mathrm{~g}$ & $1.40 \mathrm{a}$ & $10 / 4 \mathrm{fg}$ \\
\hline
\end{tabular}

The same letters in each column indicate no significant difference in the Duncan test at the 5\% possibility level. a:maximum, b:medium, c:minimum. + with -without.

in successive monthly intervals (Figure 1). The effects of storage time and the ENA on flesh firmness showed that use of this chemical in entire months caused more flesh consistency related to control treatment. The highest and lowest mean firmness values, $20 / 8$ and $5 / 8 \mathrm{Kg} / \mathrm{cm}^{2}$, were registered at the end of first and fifth month of storage, relatively in presence and lack of ENA (Table 5).

\subsection{Fruit Biochemical Traits}

The mean comparisons of storage period and cultivar interactions effects on the levels of $\mathrm{pH}$, TA and TSS confirmed that $\mathrm{pH}$ levels increased over time between the two cultivars which their TA values were inversely changed, decreasing during storage time, either significantly different $(p<0.05)$. The total amount of dissolved solids over time, demonstrated increasing trend both in "Wealthy" and "Dirras-e Mashhad" (Figures 2-4). Interaction between storage time and 1-MCP 
Table 3. Mean comparisons of cultivar and 1-MCP interaction on qualitative characteristics of the two apple cultivars during storage.

\begin{tabular}{|c|c|c|c|c|c|c|c|}
\hline Storage time & Cultivar & 1-MCP & $\mathrm{pH}$ & Acidity & TSS & Weight Loss & Fmax \\
\hline \multirow[t]{3}{*}{ Starting } & Dirras-e Mashhad & - & $4.56 \mathrm{a}$ & $2.96 \mathrm{n}$ & $9.6 \mathrm{k}$ & & $19.07 \mathrm{c}$ \\
\hline & Wealthy & - & $4.03 \mathrm{f}$ & $4.7 \mathrm{a}$ & $11 \mathrm{j}$ & & $19.05 \mathrm{c}$ \\
\hline & Dirras-e Mashhad & - & $4.38 \mathrm{bc}$ & $3.35 \mathrm{ij}$ & $15.5 \mathrm{~d}$ & $0.30 \mathrm{ab}$ & $28.4 \mathrm{a}$ \\
\hline \multirow[t]{4}{*}{1 st month } & & + & $4.27 \mathrm{~d}$ & $3.88 \mathrm{ed}$ & $16.06 \mathrm{c}$ & $0.11 \mathrm{ab}$ & $25.2 \mathrm{~b}$ \\
\hline & Wealthy & - & $4.55 \mathrm{a}$ & $3.95 \mathrm{~cd}$ & $13.5 \mathrm{~g}$ & $0.37 \mathrm{ab}$ & $14.6 \mathrm{~d}$ \\
\hline & & + & $4.05 \mathrm{f}$ & $4.15 \mathrm{~b}$ & $13 \mathrm{~h}$ & $0.71 \mathrm{ab}$ & $12.9 \mathrm{de}$ \\
\hline & Dirras-e Mashhad & - & $4.43 \mathrm{~b}$ & $2.85 \mathrm{o}$ & $16 c$ & $0.42 \mathrm{ab}$ & $17.8 \mathrm{c}$ \\
\hline \multirow[t]{4}{*}{ 2nd month } & & + & $4.27 \mathrm{~d}$ & $3.4 \mathrm{hi}$ & $17.1 \mathrm{a}$ & $0.32 \mathrm{~b}$ & $14.6 \mathrm{~d}$ \\
\hline & Wealthy & - & $4.07 \mathrm{f}$ & $3.5 \mathrm{~h}$ & $12.5 \mathrm{i}$ & $0.03 \mathrm{ab}$ & 10.9 ef \\
\hline & & + & $4.07 \mathrm{f}$ & $3.66 \mathrm{j}$ & $12.6 \mathrm{i}$ & $0.91 \mathrm{a}$ & $9.7 \mathrm{f}$ \\
\hline & Dirras-e Mashhad & - & $4.35 \mathrm{c}$ & $3.25 \mathrm{jk}$ & $17.4 \mathrm{a}$ & $0.27 \mathrm{ab}$ & $11.7 \mathrm{ef}$ \\
\hline \multirow[t]{4}{*}{ 3rd month } & & + & $4.41 \mathrm{~b}$ & $2.83 \mathrm{o}$ & $16.3 \mathrm{c}$ & $0.4 \mathrm{ab}$ & $6.1 \mathrm{~g}$ \\
\hline & Wealthy & - & $3.96 \mathrm{~g}$ & $3.7 \mathrm{fg}$ & $12.3 \mathrm{i}$ & $0.29 \mathrm{ab}$ & $4 \mathrm{~g}$ \\
\hline & & + & $4.06 \mathrm{f}$ & $3.98 \mathrm{~cd}$ & $12.3 \mathrm{i}$ & $0.13 \mathrm{ab}$ & $3 / 9 \mathrm{~g}$ \\
\hline & Dirras-e Mashhad & - & $4.38 \mathrm{bc}$ & $3 \mathrm{~nm}$ & $16.6 \mathrm{~b}$ & $0.29 \mathrm{ab}$ & $27 a b$ \\
\hline \multirow[t]{4}{*}{ 4th month } & & + & $4.41 \mathrm{bc}$ & $3.2 \mathrm{lk}$ & $16 c$ & $0.39 \mathrm{ab}$ & $24.8 \mathrm{~b}$ \\
\hline & Wealthy & - & $4.09 \mathrm{f}$ & $3.93 \mathrm{~d}$ & $15 \mathrm{~d}$ & $0.35 \mathrm{ab}$ & $11.8 \mathrm{ef}$ \\
\hline & & + & $4.05 \mathrm{f}$ & $3.8 \mathrm{fe}$ & $13.6 \mathrm{~g}$ & $0.22 \mathrm{ab}$ & 11.7 ef \\
\hline & Dirras-e Mashhad & - & $4.18 \mathrm{e}$ & $3.1 \mathrm{~lm}$ & $17.4 \mathrm{a}$ & $0.65 \mathrm{ab}$ & $19 \mathrm{c}$ \\
\hline \multirow[t]{3}{*}{ 5th month } & & + & $4.25 \mathrm{~d}$ & $2.66 \mathrm{p}$ & $15.5 \mathrm{~d}$ & $0.32 \mathrm{ab}$ & $20.2 \mathrm{c}$ \\
\hline & Wealthy & - & $3.74 \mathrm{~h}$ & $3.35 \mathrm{ji}$ & $14.1 \mathrm{f}$ & $0.95 \mathrm{a}$ & $10.4 \mathrm{ef}$ \\
\hline & & + & $3.75 \mathrm{~h}$ & $4.06 \mathrm{bc}$ & $14.9 \mathrm{e}$ & $0.92 \mathrm{a}$ & $8.8 \mathrm{f}$ \\
\hline
\end{tabular}

The same letters in each column indicate no significant difference in the Duncan test at the 5\% probability level. a:maximum, b:medium, c:minimum. +with -without.

Table 4. Effect of 1-MCP and ENA applications and cultivar interaction on qualitative characteristics of the two apple cultivars.

\begin{tabular}{|c|c|c|c|c|c|c|c|}
\hline Cultivar & ENA & 1-MCP & Weight Loss & $\mathrm{pH}$ & Acidity & TSS Brix & Fmax \\
\hline \multirow{4}{*}{$\begin{array}{l}\text { Dirras-e } \\
\text { Mashhad }\end{array}$} & - & - & $0.39 \mathrm{ab}$ & $4.36 \mathrm{a}$ & $3 \mathrm{e}$ & $14.8 \mathrm{~d}$ & $20.6 \mathrm{a}$ \\
\hline & - & + & $0.33 \mathrm{ab}$ & $4.36 \mathrm{a}$ & $3.12 \mathrm{e}$ & $15.7 \mathrm{c}$ & $18.1 \mathrm{~b}$ \\
\hline & + & - & $0.38 \mathrm{ab}$ & $4.36 \mathrm{a}$ & $3.11 \mathrm{e}$ & $17.3 \mathrm{a}$ & $20.8 \mathrm{a}$ \\
\hline & + & + & $0.03 \mathrm{~b}$ & $4.28 \mathrm{~b}$ & $3.27 \mathrm{~d}$ & $16.7 \mathrm{~b}$ & $18.2 \mathrm{~b}$ \\
\hline \multirow{4}{*}{ Wealthy } & - & - & $0.25 \mathrm{ab}$ & $4.14 \mathrm{c}$ & $3.83 \mathrm{~b}$ & $13.3 \mathrm{f}$ & $11.7 \mathrm{c}$ \\
\hline & - & + & $0.40 \mathrm{ab}$ & $4 \mathrm{~d}$ & $3.78 \mathrm{~b}$ & $13.5 \mathrm{e}$ & $9.6 \mathrm{~d}$ \\
\hline & + & - & $0.54 \mathrm{ab}$ & $4 \mathrm{~d}$ & $3.71 \mathrm{c}$ & $13.2 \mathrm{f}$ & $10.5 \mathrm{~cd}$ \\
\hline & + & + & $0.76 \mathrm{a}$ & $3.99 \mathrm{~d}$ & $4 \mathrm{a}$ & $13.1 \mathrm{f}$ & $9.2 \mathrm{~d}$ \\
\hline
\end{tabular}

The same letters in each column indicate no significant difference in the Duncan test at the 5\% possibility level. a:maximum, b:medium, c:minimum. +with -without. 
Table 5. Effect of 1-MCP and ENA applications during storage on qualitative characteristics of the two apple cultivars.

\begin{tabular}{|c|c|c|c|c|c|c|c|}
\hline Storage time & ENA & 1-MCP & $\mathrm{pH}$ & Acidity & TSS Brix & Weight Loss & Fmax \\
\hline \multirow[t]{2}{*}{ Starting } & - & - & $4.3 \mathrm{~cd}$ & $3.8 \mathrm{~b}$ & $10.3 \mathrm{~g}$ & & $19 / 6 \mathrm{ab}$ \\
\hline & - & - & $4.5 \mathrm{a}$ & $3.7 \mathrm{c}$ & $15 \mathrm{c}$ & $0.38 \mathrm{a}$ & $21.3 \mathrm{a}$ \\
\hline \multirow[t]{4}{*}{ 1st month } & - & + & $4.1 \mathrm{gh}$ & $4 \mathrm{a}$ & $14 \mathrm{e}$ & $0.36 \mathrm{a}$ & $18.2 \mathrm{bc}$ \\
\hline & + & - & $4.3 \mathrm{~b}$ & $3.6 \mathrm{cde}$ & $14 \mathrm{e}$ & $0.29 \mathrm{a}$ & $21.8 \mathrm{a}$ \\
\hline & + & + & $4.1 \mathrm{fgh}$ & $3.9 \mathrm{a}$ & $15 \mathrm{c}$ & $0.46 \mathrm{a}$ & $19.9 \mathrm{ab}$ \\
\hline & - & - & $4.2 \mathrm{cde}$ & $2.9 \mathrm{k}$ & $13 \mathrm{f}$ & $0.21 \mathrm{a}$ & $14.2 \mathrm{ef}$ \\
\hline \multirow[t]{4}{*}{ 2nd month } & - & + & $4.1 \mathrm{gh}$ & $3.5 \mathrm{def}$ & $15.1 \mathrm{bc}$ & $0.39 \mathrm{a}$ & $11.8 \mathrm{f}$ \\
\hline & + & - & $4.2 \mathrm{def}$ & $3.4 \mathrm{gh}$ & $15.5 \mathrm{~b}$ & $0.24 \mathrm{a}$ & $14.5 \mathrm{ef}$ \\
\hline & + & + & $4.1 \mathrm{efgh}$ & $3.5 \mathrm{def}$ & $14.6 \mathrm{~d}$ & $0.19 \mathrm{a}$ & $12.5 \mathrm{f}$ \\
\hline & - & - & $4.1 \mathrm{efgh}$ & $3.4 \mathrm{fgh}$ & $14.4 \mathrm{~d}$ & $0.25 \mathrm{a}$ & $7.1 \mathrm{gh}$ \\
\hline \multirow[t]{4}{*}{ 3rd month } & - & + & $4.3 \mathrm{bc}$ & $3.1 \mathrm{j}$ & $14.3 \mathrm{ed}$ & $0.28 \mathrm{a}$ & $4.4 \mathrm{i}$ \\
\hline & + & - & $4.1 \mathrm{~h}$ & $3.5 \mathrm{efg}$ & $15.3 \mathrm{bc}$ & $0.30 \mathrm{a}$ & $8.6 \mathrm{~g}$ \\
\hline & + & + & $4.1 \mathrm{gh}$ & $3.6 \mathrm{~cd}$ & $14.3 \mathrm{de}$ & $0.25 \mathrm{a}$ & $5.6 \mathrm{hi}$ \\
\hline & - & - & $4.2 \mathrm{cde}$ & $3.5 \mathrm{def}$ & $15.5 \mathrm{~b}$ & $0.34 \mathrm{a}$ & $21.3 \mathrm{a}$ \\
\hline \multirow[t]{4}{*}{ 4th month } & - & + & $4.2 \mathrm{cde}$ & $3.6 \mathrm{~cd}$ & $15.1 \mathrm{bc}$ & $0.37 \mathrm{a}$ & $19.3 \mathrm{ab}$ \\
\hline & + & - & $4.2 \mathrm{efg}$ & $3.3 \mathrm{ghi}$ & $16.1 \mathrm{a}$ & $0.30 \mathrm{a}$ & $17.4 \mathrm{bc}$ \\
\hline & + & + & $4.2 \mathrm{efg}$ & $3.3 \mathrm{hi}$ & $14.5 \mathrm{~d}$ & $0.25 \mathrm{a}$ & $17.2 \mathrm{bcd}$ \\
\hline & - & - & $3.9 \mathrm{j}$ & $3.3 \mathrm{i}$ & $16.1 \mathrm{a}$ & $0.43 \mathrm{a}$ & $13.3 \mathrm{ef}$ \\
\hline \multirow[t]{3}{*}{ 5th month } & - & + & $4 \mathrm{i}$ & $2.8 \mathrm{j}$ & $14.4 \mathrm{ed}$ & $0.43 \mathrm{a}$ & $15.6 \mathrm{cde}$ \\
\hline & + & - & $3.9 \mathrm{ij}$ & $3.1 \mathrm{j}$ & $15.4 \mathrm{~b}$ & $1.17 \mathrm{a}$ & $16 \mathrm{cde}$ \\
\hline & + & + & $3.9 \mathrm{ij}$ & $3.8 \mathrm{~b}$ & $16 \mathrm{a}$ & $0.81 \mathrm{a}$ & $13.5 \mathrm{ef}$ \\
\hline
\end{tabular}

The same letters in each column indicates no significant difference in the Duncan test at the 5\% possibility level. a:maximum, b:medium, c:minimum. + with -without.

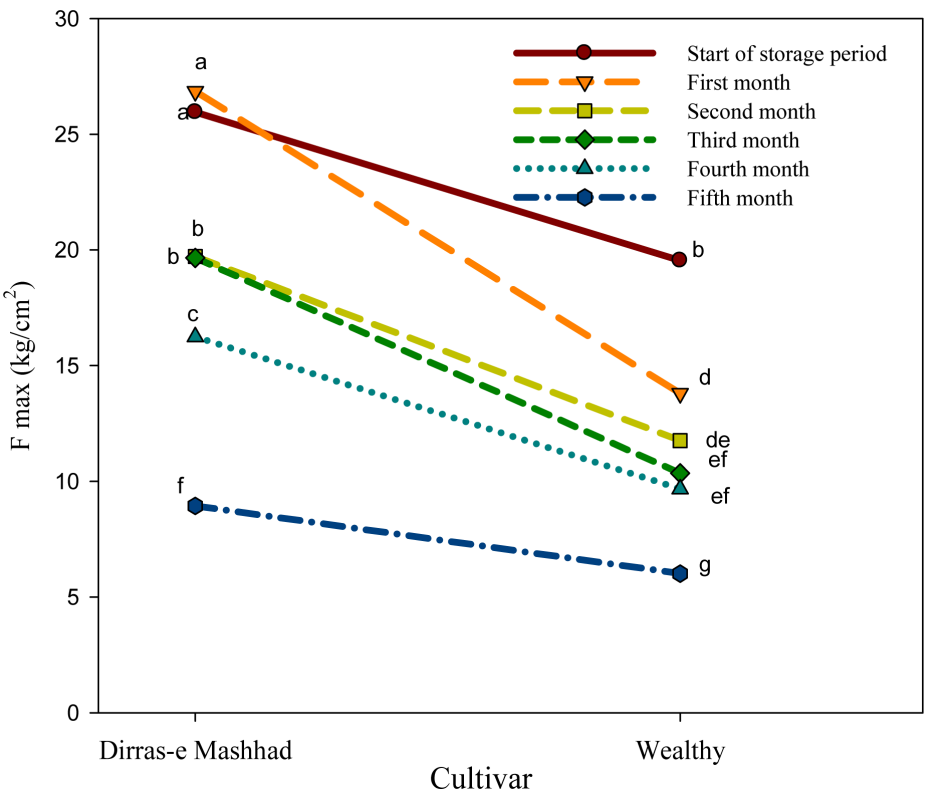

Figure 1. Mean comparisons effects of interaction between storage time and cultivar on flesh firmness of apples. 


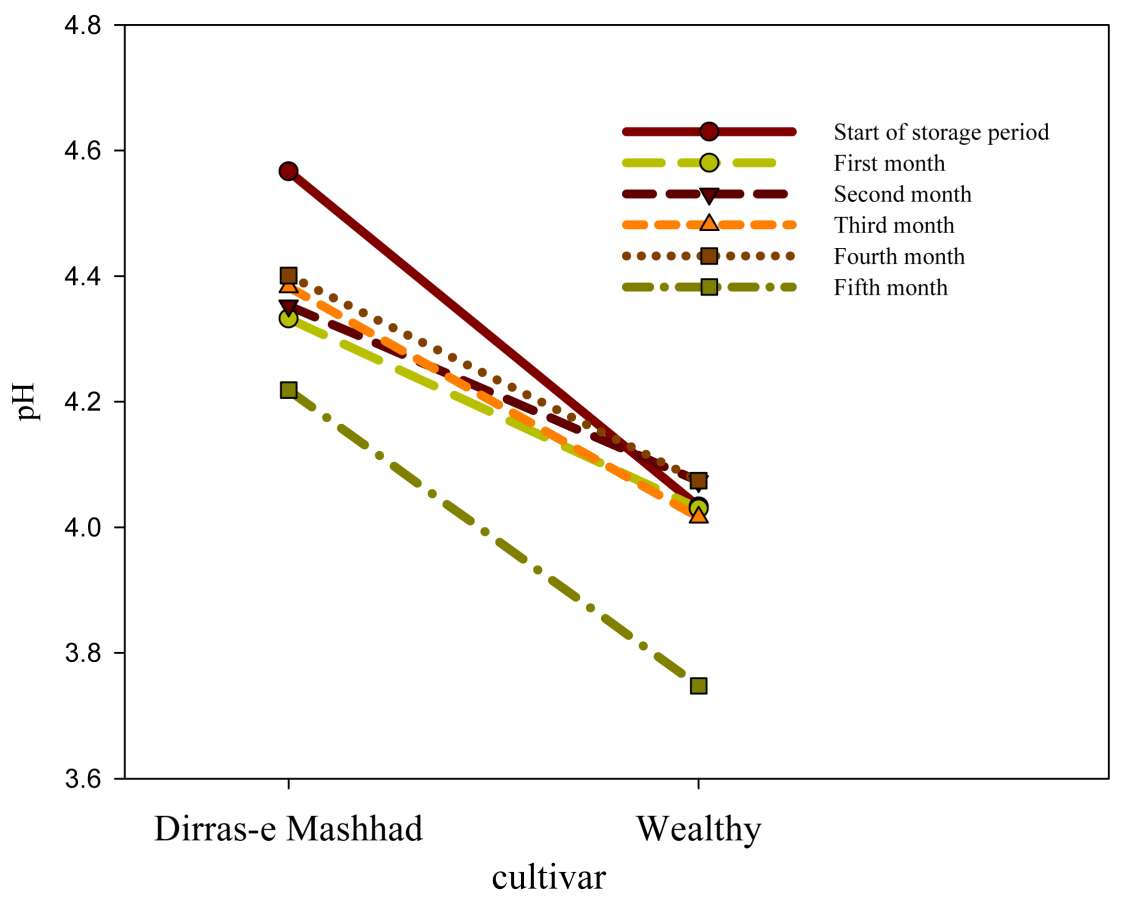

Figure 2. Mean comparisons effects of interaction between cultivar and storage period on $\mathrm{pH}$ of apples.

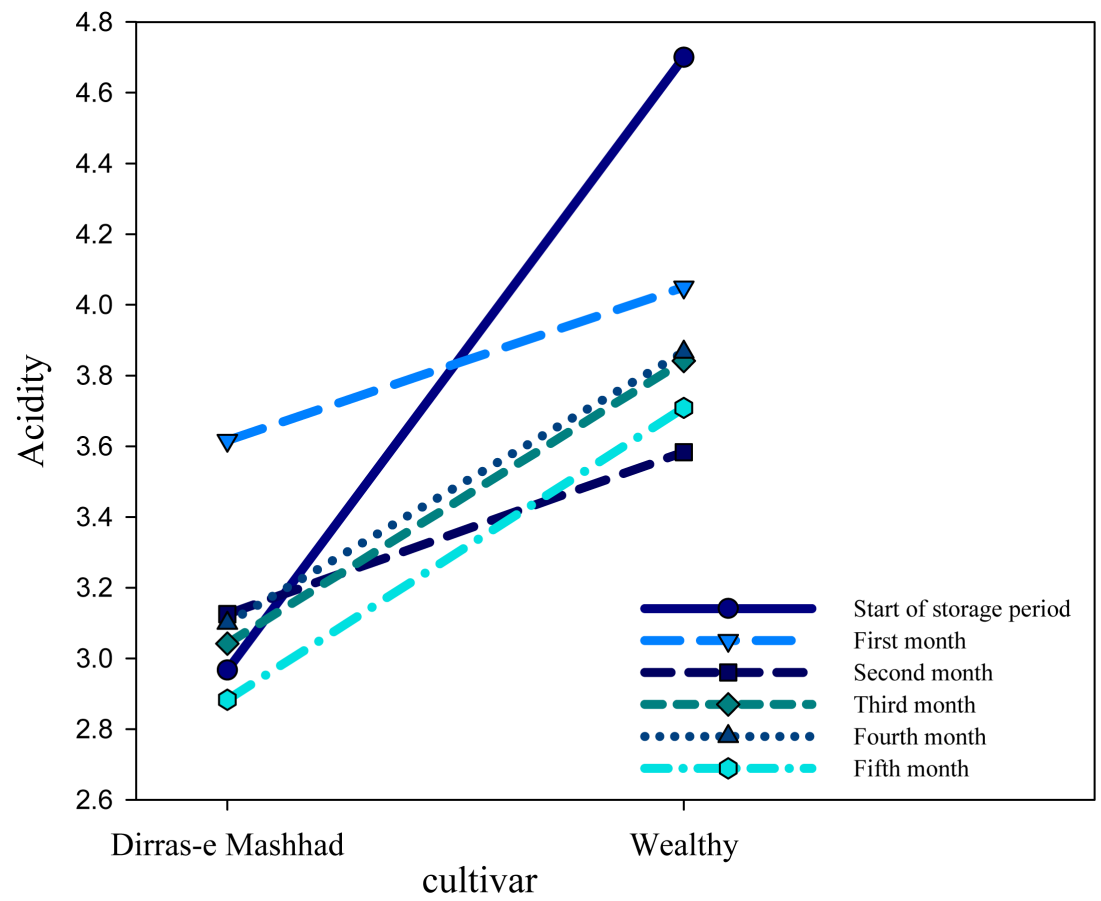

Figure 3. Mean comparisons effects of interactions between cultivar and storage time on TA of apples.

on TA, $\mathrm{pH}$ and TSS showed that use of 1-MCP affected significantly $\mathrm{pH}$ of juice ( $\mathrm{p}<0.05$ ), inhibiting it to increase. Vice verse, 1-MCP influenced TA significantly $(\mathrm{p}<0.05)$ preventing its decrement. Use of 1-MCP affected equally TSS by increasing values (Figures 5-7). Mean comparisons of interaction effects of 


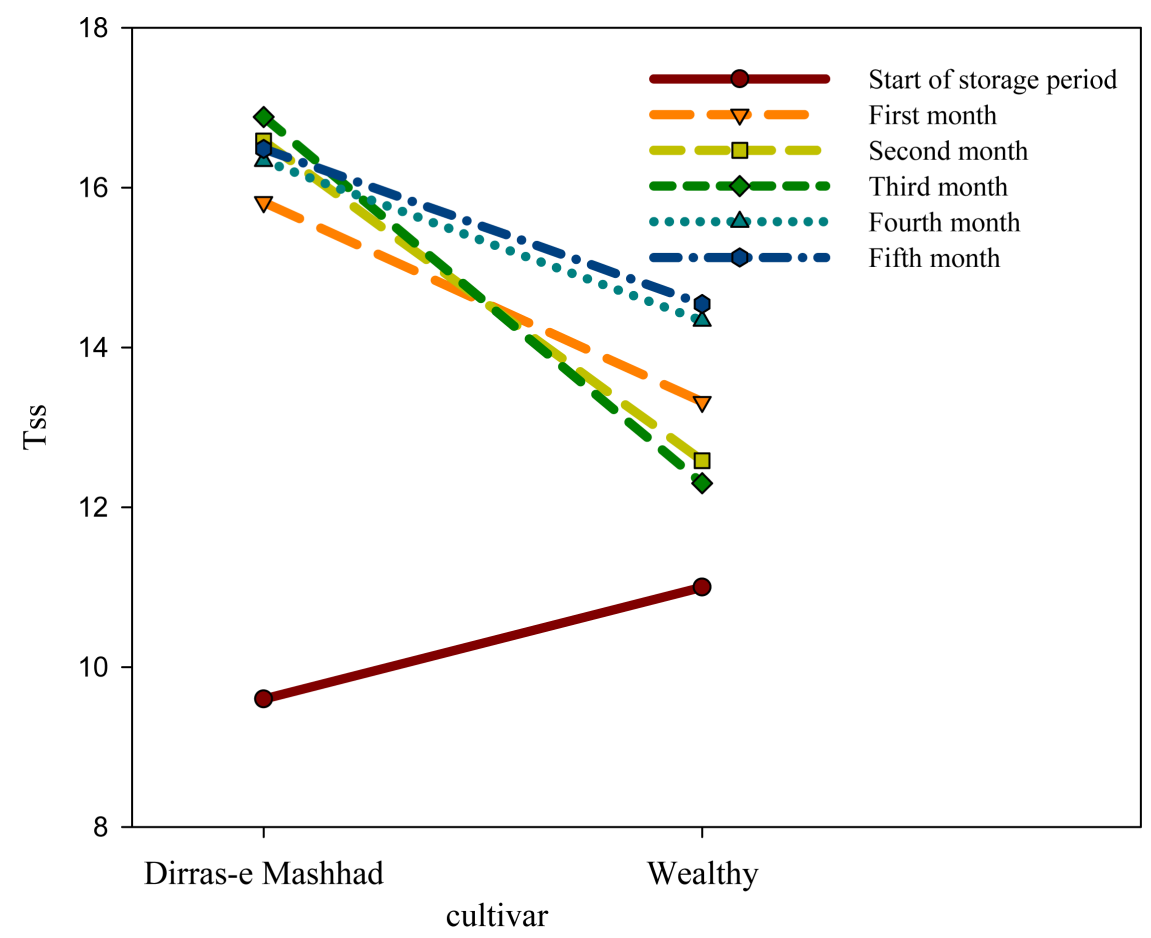

Figure 4. Mean comparisons effects of interaction between cultivar and storage time on TSS of apples.

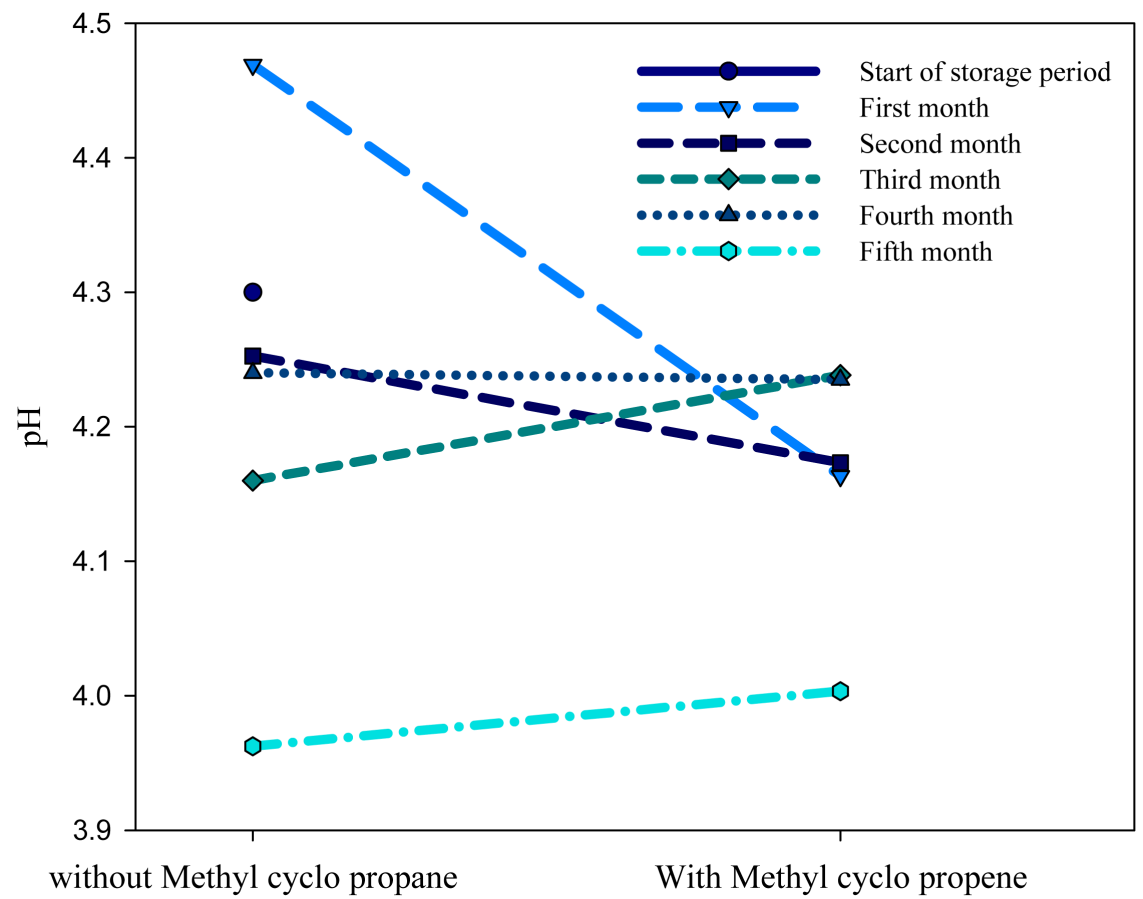

Figure 5. Mean comparisons effects of interaction between 1-MCP and storage time on the $\mathrm{pH}$ of apples.

ENA, cultivar and TSS sustained that the highest TSS value was obtained by ENA treatment in "Dirras-e Mashhad" and the lowest in "wealthy" (Table 6). ENA, 1-MCP and TSS mean comparisons of interaction effects endorsed that the 


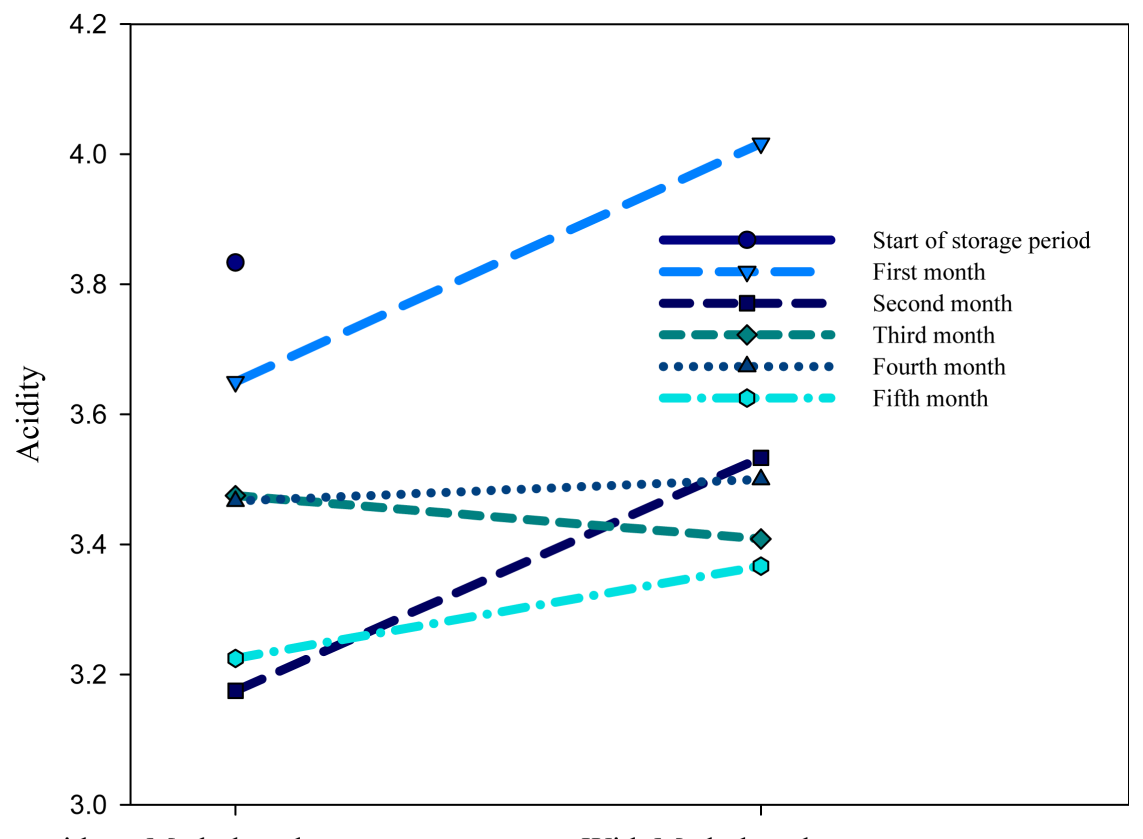

without Methyl cyclo propane

With Methyl cyclo propene

Figure 6. Mean comparisons effects of interaction between 1-MCP and storage time on TA of apples.

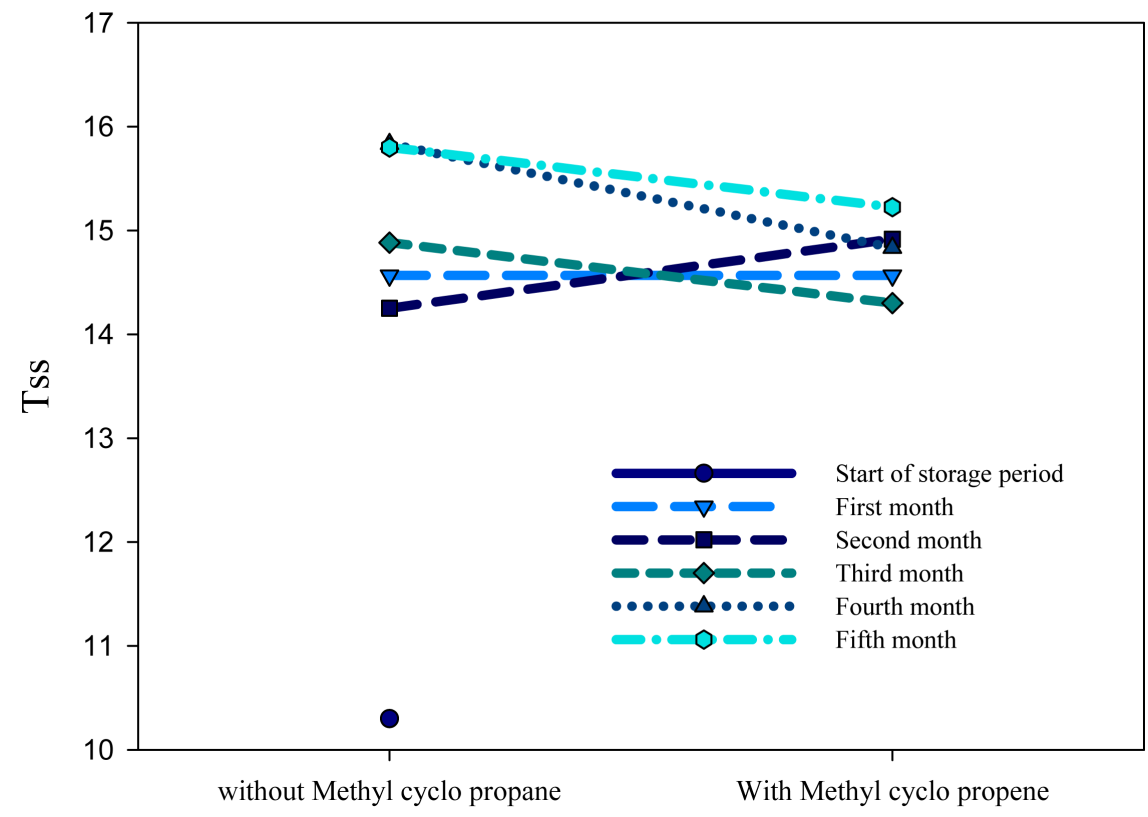

Figure 7. Mean comparisons effects of interaction between 1-MCP and storage time on TSS of apples.

Table 6. Mean comparisons effects of ENA and cultivar interaction on TSS of apples.

\begin{tabular}{ccc}
\hline Cultivar & ENA & without ENA \\
\hline Dirras-e Mashhad & $16.36 \mathrm{a}$ & $15.23 \mathrm{~b}$ \\
Wealthy & $12.97 \mathrm{c}$ & $13.4 \mathrm{c}$ \\
\hline
\end{tabular}

The same letters in each column indicate no significant difference in the Duncan test at the $5 \%$ possibility level. a:maximum, b:medium, c:minimum. 
highest and the lowest of ethylene absorption is respectively related to use of ENA and non that of 1-MCP. Ethylene absorption was improved by ENA, and ENA+1-MCP treatments affecting mean values of 15.3 and 14.91 of TSS, correspondingly (Table 7).

\subsection{Sensory Characteristics}

Application of sole ENA improved general acceptability of "Dirras-e Mashhad"'s at maximum level and the lowest percentage of acceptance through combined treatment of ENA+1-MCP. Contradictory responses were registered in terms of aroma, the maximum level of ENA treated samples was found in "Dirras-e Mashhad", while the minimum rates of aroma was noted in 1-MCP treated samples of "wealthy", combined use of ENA+1-MCP and non-treated (control) treatments of "Dirras-e Mashhad". Furthermore, single treatments showed high acceptance rates in "Dirras-e Mashhad", the cultivar that gained higher scores for each single panel characteristics when treated by ENA. Instead, the non treated samples, as control, led to least scores for fruit taste (Table 8). Others also noticed the that 1-MCP treatment increased fruit firmness in "Cripps Pink" while on "Fuji" and "Gala" firmness got only slightly increased Zanella \& Rossi. 2015 [8]. "Bartlett" pear fruit ripening was assessed by exposure to concentrations of 1 -MCP as $42 \mu \mathrm{mol} \cdot \mathrm{m}^{-3}$ or $0.42 \mu \mathrm{mol} \cdot \mathrm{m}^{-3}$ at harvest and then $+4.2^{\circ}$. Treated fruits contained similar peel yellow color, TA and soluble solid concentration, but higher firmness after 180 days storage compared to control fruit after 60 days storage. Reapplication of the compound based on fruit ethylene production capacity showed to be efficient. A low dose of 1-MCP application at harvest, reapplied by higher doses assessing the rate of ethylene production, re-

Table 7. Effect of ENA and 1-MCP on the TSS of apples.

\begin{tabular}{ccc}
\hline & With 1-MCP & Without 1-MCP \\
\hline without ENA & $14.62 \mathrm{ab}$ & $14.1 \mathrm{~b}$ \\
with ENA & $14.91 \mathrm{ab}$ & $15.3 \mathrm{a}$ \\
\hline
\end{tabular}

The same letters in each column indicate no significant difference in the Duncan test at the 5\% possibility level. a:maximum, b:medium, c:minimum.

Table 8. Mean comparison effects of ENA, 1-MCP and cultivar interaction on the sensory characteristics of apples.

\begin{tabular}{cccccc}
\hline Cultivar & ENA & $1-$ MCP & Taste (\%) & Aroma (\%) & Acceptance \\
\hline Dirras-e Mashhad & + & - & $75 \mathrm{a}$ & $73.33 \mathrm{a}$ & $70 \mathrm{a}$ \\
Dirras-e Mashhad & + & + & $33.3 \mathrm{~b}$ & $40 \mathrm{c}$ & $36.7 \mathrm{~b}$ \\
Dirras-e Mashhad & - & + & $71.7 \mathrm{a}$ & $68.3 \mathrm{ab}$ & $41.67 \mathrm{~b}$ \\
Dirras-e Mashhad & - & - & $31.67 \mathrm{~b}$ & $50 \mathrm{c}$ & $55 \mathrm{ab}$ \\
Wealthy & + & - & $51.67 \mathrm{ab}$ & $50 \mathrm{abc}$ & $50 \mathrm{ab}$ \\
Wealthy & + & + & $45 \mathrm{~b}$ & $43 \mathrm{bc}$ & $45 \mathrm{ab}$ \\
\hline
\end{tabular}

The same letters in each column indicate no significant difference in Duncan test at the 5\% level. a:maximum, b:medium, c:minimum. +with -without. 
moved from cold storage, Bartlett' pear storage life was extended (Argenta et al. 2016) [16].

\section{Discussion and Conclusion}

\subsection{Fruit Weight Loss}

Dehydration could deteriorate fruit weight and quality; the latter decayed organoleptic characteristics and also the appearance causing peel shrinkage. So during post harvest, all factors affecting the increase of the rate of fruit respiration should be controlled to preserve fruit moisture. The cultivars genetically capable of conserving longer the fruit weight are preferred (Bahari et al. 2010) [17], as it was assessed through a cold storage trial of $49 \mathrm{mid}$, late and very late ripening commercial apples, ranked in different groups. Morgan \& Richards 2002 [18] described different traits of 2000 apple cultivars including fruit storability potential. Weight loss occurs per via respiration and combustion of organic substances like sugars (Wills et al. 1998 [19], Hajnajari et al. 2010) [20]. Use of 1-MCP and ENA could reduce respiration rate and also decelerate production of ethylene biosynthesis all leading to slowing down conversion of starch into the carbohydrates all with the aim of extending storage life. Due to genetic variability, fruit peel anatomy in different cultivars recites positive role in storability by means of more protective layers of wax or thick epidermis (Veraverbeke et al. 2001) [21], the traits for which "Dirras-e Mashhad" showed less weight loss than "Wealthy". Cold storage of 7 Iranian early apple cultivars showed that, those with thin fruit peel, soft texture, juicy flesh and smaller size showed more limited storage life of 8 weeks equal to half of the dominant group (Hajnajari \& Eshghi, 2013) [22].

\subsection{Flesh Firmness}

Degradation of pectin compounds is reported as the main factor responsible for loose of flesh firmness in apple during ripening where genetic effect of cultivar showed to be significant on flesh softening. But the texture is defined also by mechanical and geometrical components including hardness, chewiness, viscosity and particle size and shape (Tromp et al. 2005) [5]. Proceeding with maturation, ripening and fruit aging and enzymes activities, cell wall polysaccharides undergo hydrolysis loosing texture integrity and hence softened flesh (Chen et al. 2006) [23]. The use of 1-MCP and NEA decelerated ethylene production, delayed the apple fruit maturity and ripening during storage. According to the reported results (Jeziorek et al. 2010 [24]; and Sardabi et al. 2014 [25]), 1-MCP treatment enhanced flesh tissue firmness of "Golden Delicious" fruits; the same response was observed on the treated samples of the apple cultivars in the present study. Newly released early apple "GolBahar" demonstrated 4 months of storability keeping eating quality because of high grade of flesh firmness, 5 $\mathrm{kg} / \mathrm{cm}^{2}$, as well (Hajnajari, 2016) [26]. These results are equally confirmed by the present study. It was deduced that besides delayed ripening, due to reduced ethylene production, it prevented rapid fruit flesh softening plus preventing any change of over color. Other flesh traits influence the cultivar role including cell 
density as number of cells per volume unit, cell wall thickness and different metabolism pathway (Johnston \& Hewett, 2002) [27], so "Dirras-e Mashhad" was marked by higher firmness than "Wealthy". The same variability for different flesh traits was registered among 29 apple cultivars stored in $+2^{\circ} \mathrm{C}$ and $95 \% \mathrm{RH}$ at $4^{\text {th }}$ month continued with a decreasing trend of flesh firmness, highest values of storability were found in "Topaz", "Fiesta" and "Fuji" relatively 50.5, 57.3 and 64.1 N (Soska \& Tomala, 2006) [28]. In agreement with our results others observed similar cultivar-dependent response regarding flesh firmness trend in the harvested fruits at three different growth stages during storage, even in the samples exposed to 1-MCP related to controlled atmosphere (CA) or low oxygen treatments. 1-MCP treated and untreated fruit samples of "Red Delicious" in both storage conditions, CA and low oxygen in comparison with normal cold storage conditions it was confirmed that the innovative techniques provide considerable storability and particularly flesh firmness (Zanella \& Rossi, 2015) [8].

\subsection{Oscillations in TSS Levels}

TSS value is a prevailed index of palatability indicating the amount of converted starch principally in sugars, albeit as a maturity index Tromp et al. 2005 [5]. Surveys performed on the average level of TSS during storage indicated that storing the fruit over longer times, sugar level will increase. Harvested fruits of "Shampion", 5 times at weekly intervals at more stages of fruit growth, during and after predictable optimum harvest date, till physiological maturity or ripening, using starch index approved that quality parameters will change according to harvest date. So, late harvested samples overtook by increased TSS values could shorten the storability (Kvikliene \& Valiuškaitè, 2009) [29], nevertheless, others reported the reduction of TSS during storage (Naseri et al., 2001) [30]. Even some results regarding 1-MCP treatment indicate no significant effect ( $\mathrm{p}<$ 0.05) on TSS (De Filippi et al. 2004 [31]; Moran \& McManus, 2005 [32] [33]) but use of NAE and control treatments (normal pathway) both increased the TSS levels during storage. Negative correlation was found between TSS and fruit weight studying pomologies in 8 promising apple genotypes. IRI6 and IRI1 showed relatively 13 and 18.5 of grade (Brix Zand \& Hajnajari, 2011) [34]. Timing and 1-MCP reapplication concentration ought to be controlled for desired quality, TA, SSC, flesh firmness as well as physiological disorders Argenta et al. 2016 [16].

\subsection{Acidity and Fruit $\mathrm{pH}$}

Mean comparisons of $\mathrm{pH}$ and $\mathrm{TA}$ revealed that in the long run $\mathrm{pH}$ increased but TA decreased, alterations caused by malic acid production due to respiratory activities. As a rule, "to lower descends the TA, the higher level will ascend the $\mathrm{pH}^{\prime}$, and since organic acids as the raw materials are used during respiration, reduced acidity and increased $\mathrm{pH}$ occurs during storage, so more organic acids will convert to glucose (Shaykh-al-Eslami, 2004) [35]. Others noted TA level comes down during fruit development stages till ripening; the process continued 
either during storage period. Such trend of TA decline acceleration is predominantly influenced by genetic specification of the cultivar (Soska \& Tomala, 2006) [28]. Also Sardabi et al. 2014 [25] reported 1-MCP and ethylene absorber maintained lower $\mathrm{pH}$ values comparing to untreated apples. In treated apples by 1-MCP and stored under controlled atmosphere, the main event in fruits was the TA reduction level, stored under different conditions. In accordance with the mentioned reports, the TA diminished in both cultivars in exam even with differences during storage, but disaccording with them, the use of 1-MCP and NEA impeded the $\mathrm{pH}$ to increase (Akbudak et al. 2009) [36].

\subsection{Sensory Characteristics}

Generally, fruits at ripening stage would find the desired aroma and flavor. Moreover, cultivars differ from each other in ripening time to achieve the desired sensory properties 9Pelayo-zaldivar, 2005) [4]. The panel group concluded that native "Dirras-e Mashhad" contained better inherent aroma composed of innumerable volatile compounds such as derivates of alcohol, aldehydes, carboxylic esters, ketones and ether, properties decisively depending to the genetics compared with "Wealthy", both grown under identical orchard management and climatic conditions. The observed differences between treatments related to control, affirm the relative efficiencies of the proposed chemicals on qualities and weights of apples. It was shown that use of 1-MCP and ENA could increase the period of qualified cold storage, depending also on the cultivar. The highest flesh stiffness was held at 5th month by ENA, and the least weight loss was achieved by 1-MCP. Other noticeable traits may be defined as flesh firmness, TSS, TA and improved over-color. Genetic factor played prominent role in de/accelerating the alterations. Since those cultivars like Dirras-e Mashhad that suffer less alteration in unit of time, it reflects a higher storage potential. Furthermore sole 1-MCP had significant effect on marketability and key commercial traits of fruits e.g. weight, flesh firmness and over-color intensity indicating it as an acceptable chemical to increase the apple storage time. ENA could also maintain relatively the firmness compared with control. Aroma was not affected by 1-MCP treatment neither in "Wealthy" nor in "Dirras-e Mashhad", in agreement with the other results on "Red Delicious" and "Golden Delicious" (Kondo et al. 2005) [37]. The observations regarding aroma was either reported by Mattheis et al. 2005 [38], so that 1-MCP treated "Gala" apples, stored in different conditions including CA, produced less volatile compounds than control. Others by treatment of "Granny Smith" apples with 1-MCP could extend the storage time in normal storage conditions for at least 3 months without losing freshness. Descriptive sensory analysis affirmed that 1-MCP treated samples suffered low rates loss of juiciness, cohesiveness, hardness, crunchiness, greenness and sourness during storage as compared to control. All of the samples appeared on the positive side of 'Freshness' axis in principal component space. The highest level of freshness loss during the storage period was observed in control samples, which suffered changes in quality after the 9 months of storage, so that the fruit 
were totally decayed (Tomic et al. 2016) [9].

\subsection{Ethylene Production}

The analysis of variance for fruit ethylene emission showed significant difference only in storage time. The mean comparisons of ethylene production at initial time of experiment and $5^{\text {th }}$ month of storage measured relatively at 3.26 and $53.64 \mathrm{ppm} / \mathrm{Kg} \cdot \mathrm{hr}$. The assessments emphasize the increasing trend of ethylene production correlated directly with respiration of the fruit senescence reflected in cell wall softening due to enzymatic action. Occurrence of known apple disorders such as scald, bitter pit and internal browning during storage all go through the similar metabolic pathway for which the final reaction lead to stimulation and production of ethylene (Tromp et al. 2005) [5].

\section{7. $\mathrm{CO}_{2}$ Production}

The analysis of variance for fruit $\mathrm{CO}_{2}$ production afforded the significant differences regarding storage period, cultivar, interactions of time $\times$ cultivar, time $\times$ cultivar $\times 1 \mathrm{MCP}$ and time $\times \mathrm{KMnO}_{4} \times 1 \mathrm{MCP}$, as well. No significant difference was noted for other traits. The mean comparisons of emitted $\mathrm{CO}_{2}$ during storage confirmed that the least value 2301.6 was recorded for initial stage of storage, but it in an increasing trend it grows to $2610.1,3085.2$ and $3154.6 \mathrm{ppm} / \mathrm{kg} \cdot \mathrm{h}$ in monthly intervals, first to $3 \mathrm{rd}$ month of storage, with a slight reduction (3115.3) at the end of 4th month and a noticeable decline (2592.5) at final stage of storage. There was observed significant difference of generated $\mathrm{CO}_{2}$ at 5 percent of probability between the first 30 days of storage and other consecutive 90 days. The mean comparisons of produced $\mathrm{CO}_{2}$ by the two apple cultivars showed significant difference during entire period of storage. "Wealthy" with the mean

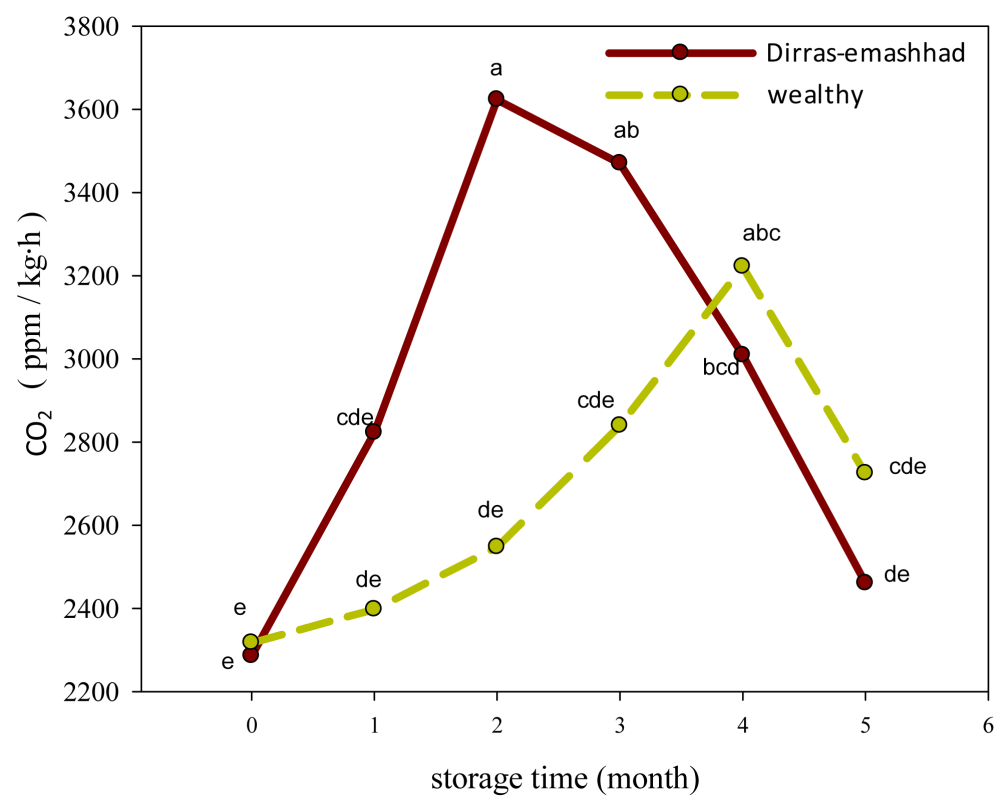

Figure 8. Mean comparisons effects of interaction between cultivar and storage time on the $\mathrm{CO}_{2}$ emission (ppm/kg.h) of apples. 
value of $36.5 \mathrm{ppm} / \mathrm{kg} \cdot \mathrm{h}$ produced more $\mathrm{CO}_{2}$ than "Dirras-e Mashhad" that emitted $34.16 \mathrm{ppm} / \mathrm{kg} \cdot \mathrm{h}$. Interactions between time $\times$ cultivar on generated $\mathrm{CO}_{2}$ demonstrated that over time $\mathrm{CO}_{2}$ production was gradually increased so that the highest rates were observed in "Wealthy" and "Dirras-e Mashhad", 2839.2 and $3623 \mathrm{ppm} / \mathrm{kg} . \mathrm{h}$ during the third and second month, while the least values 2317.3 and 2285.8 were registered at initial stage of storage, correspondingly, confirming the "Wealthy" with highest respiration rate. At the end of fifth month both of cultivars decreased the emission of $\mathrm{CO}_{2}$ (Figure 8).

\section{Acknowledgements}

We acknowledge Agricultural Engineering Research Institute for Instrumental laboratory aids and Temperate Fruit Research Institute for providing the plant genetic resources maintained in National Collection of Native and Imported Commercial Apple Cultivars in Karaj, Iran.

\section{References}

[1] FAO Statistical Year Book (2012). http://www.fao.org/economic/ess/ess-publications/ess-yearbook/yearbook2012/en/

[2] Harker, F.R., Gunson, F.A. and Jaeger, S.R. (2003) The Case for Fruit Quality: An Interpretive Review of Consumer Attitudes and Preferences for Apples. Postharvest Biology and Technology, 28, 333-347. https://doi.org/10.1016/S0925-5214(02)00215-6

[3] Rahemi, M. (2004) An Introduction on Physiology and Handling of Fruit, Vegetables and ornamentals Postharvest. Shiraz University Press, Shiraz.

[4] Pelayo-Zaldivar, C., Ebeler, S.E. and Kader, A.A. (2005) Cultivar and Harvest Date Effects on Flavor and Other Quality Attributes of California Strawberries. Journal of Food Quality, 28, 78-97. https://doi.org/10.1111/j.1745-4557.2005.00005.x

[5] Tromp, J., Webster, A.D. and Wertheim, S.J. (2005) Fundamentals of Temperate Zone Tree Fruit Production. Backhuys Publishers, Leiden, 400 p.

[6] Sharples, R.O. and Johnson, D.S. (1986) Effects of Some Growth Regulators on the Ripening and Storage Quality of Apples and Pears. ActaHorticulturae, 179, 721-730. https://doi.org/10.17660/actahortic.1986.179.124

[7] JaliliMarandi, R. (2008) Postharvest Physiology. Jahade Keshavarzi, Urmia. Iran, $276 \mathrm{p}$.

[8] Zanella, A. and Rossi, O. (2015) Post-Harvest Retention of Apple Fruit Firmness By1-Methylcyclopropene (1-MCP) Treatment or Dynamic CA Storage with Chlorophyll Fluorescence (DCA-CF). European Journal of Horticultural Science, 80, 11-17. https://doi.org/10.17660/eJHS.2015/80.1.2

[9] Tomic, N., Radivojevicb, D., Milivojevicb, J., Djekic, I. and Smigic, N. (2016) Effects of 1-Methylcyclopropene and Diphenylamine on Changes in Sensory Properties of "Granny Smith" Apples during Postharvest Storage. Postharvest Biology and Technology, 112, 233-240. https://doi.org/10.1016/j.postharvbio.2015.09.009

[10] Zomorrodi, N. (2005) Effect of Packing Sort of Potassium Permanganate on the Quality of Packaging and Storage Shelf Life in Three Cultivars of Apples in Cold Storage. Journal of Agricultural Engineering, 24, 156-143.

[11] Finny, E.E. and Massie, D.R. (1975) Instrumentation for Testing the Response of Fruits to Mechanical Impact. Transactions of the ASAE, 18, 1184-1187. 
https://doi.org/10.13031/2013.36766

[12] Pre-Aymard, C., Fallik, E., Weksler, A. and Lurie, S. (2005) Sensory Analysis and Instrumental Measurements of "Anna" Apples Treated with 1-Methylcyclopropene. Postharvest Biology and Technology, 36, 135-142. https://doi.org/10.1016/j.postharvbio.2004.12.007

[13] Watkins, C.B. (2003) Principles and Practices of Postharvest Handling and Stress. In: Ferree, D.C. and Warrington, I.J., Eds., Apples. Botany, Production and Uses, CABI Publishing, Oxford, 585-614. https://doi.org/10.1079/9780851995922.0585

[14] Hosseini, S. (1990) Methods in Food Analysis. Shiraz University Press, Shiraz, 210 p.

[15] DeElla, J.R., Murrb, D.S., Murray, P., Porteousc, D. and Rupasingheb, H.P.V. (2002) Influence of Temperature and Duration of 1-Methylcyclopropene (1-MCP) Treatment on Apple Quality. Postharvest Biology and Technology, 24, 349-335. https://doi.org/10.1016/S0925-5214(01)00136-3

[16] Argenta, L.C., Mattheis, J.P., Xuetong, F., Cassandro, V. and Amarante, T. (2016) Managing "Bartlett" Pear Fruit Ripening with 1-Methylcyclopropene Reapplication during Cold Storage. Postharvest Biology and Technology, 113, 125-130. https://doi.org/10.1016/j.postharvbio.2015.11.009

[17] Bahari, A., Hajnajari, H., Kalantari, S., Rasuli, V. and Damyar, S. (2010) Grouping of 49 Iranian and Imported Apple Genotypes. Proceedings of the International Scientific Conference of Fruit Growing Intensification in Belarus: Traditions, Progress, Prospects, Belarus, 2010, 40-42.

[18] Morgan, J. and Richards, A. (2002) The New Book of Apples. Ebury Press, London, $316 \mathrm{p}$.

[19] Wills, R., McGlasson, B., Graham, D. and Joyce, A.D. (1998) Postharvest Handling of fruit, Vegetables Ornamentals. Cab International, Wallingford.

[20] Hajnajari, H., Koochaki, M.F. and Peyghambari, A. (2010) Investigation on Cold Storage Capacity of Early and Mid Ripening Apple Cultivars of Iran. Acta Horticulturae, 877, 905-910. https://doi.org/10.17660/ActaHortic.2010.877.121

[21] Veraverbeke, E.A., Lammertyn, J., Saevels, S. and Nicolai, B.M. (2001) Changes in Chemical Wax Composition of Three Different Apple (Malus domestica Borkh.) Cultivars during Storage. Postharvest Biology and Technology, 23, 197-208. https://doi.org/10.1016/S0925-5214(01)00128-4

[22] Hajnajari, H. and Eshghi, M. (2013) Influence of Harvest Time on Some Sensorial Parameters of Some Summer Apples during Cold Storage. Horticultural Sciences, 27, 275-585.

[23] Chen, J.L., Yan, S., Feng, Z., Xiao, L. and Hu, X.S. (2006) Changes in the Volatile Compounds, Chemical and Physical Properties of "Yali" Pear (Pyrus bertschneideri Rehd.) during Storage. Food Chemistry, 97, 248-255.

https://doi.org/10.1016/j.foodchem.2005.03.044

[24] Jeziorek, K., Woźni, A.K.M. and Tolmala, K. (2010) Response of "Golden Delicious" Apples to Postharvest Application of 1-Methylcyclopropene (1-MCP) in Conditions of Normal and Controlled Atmosphere. Journal of Fruit and Ornamental Plant Research, 18, 223-237.

[25] Sardabi, F., Mohtadinia, J., Shavakhi, F. and Ashraf Jafari, A. (2014) The Effects of 1-Methylcyclopropene (1-MCP) and Potassium Permanganate Coated Zeolite Nanoparticles on Shelf Life Extension and Quality Loss of Golden Delicious Apples. Journal of Food Processing and Preservation, 38, 2176-2182.

https://doi.org/10.1111/jfpp.12197 
[26] Hajnajari, H. (2016) Guide of Apple Cultivation with Emphasizing on Research Findings. Agriculture Nashre Amuzesh Publisher, Karaj, Iran, 416 p.

[27] Johnston, J.W. and Hewett, E.W. (2002) Postharvest Softening of Apple (Malusdomestica Borkh.) Fruit: A Review. New Zealand Journal of Crop and Horticultural Science, 30, 145-160. https://doi.org/10.1080/01140671.2002.9514210

[28] Soska, A. and Tomala, K. (2006) Internal Quality of Apples during Storage. Agronomijas Vēstis, 9, 146-151.

[29] Kvikliene, N. and Valiuškaite, A. (2009) Influence of Maturity Stage on Fruit Quality during Storage of "Shampion" Apples. Sodininkyste Ir Daržininkyste, 28, 117 123.

[30] Naseri, L., Babalar, M., Asgari, M. and Talaei, A.S. (2001) Effect of Foliar Spraying of Micronutrients and Cold Storage on the Quality of Golden Delicious Apple Fruit. Journal of Agricultural Science, 36, 591-597.

[31] DeFilippi, B.G., Dandekar, A.M. and Kader, A.A. (2004) Impact of Suppression of Ethylene Action or Biosynthesis on Flavor Metabolites in Apple (Malus domestica Borkh.) Fruits. Journal of Agricultural and Food Chemistry, 52, 5694-5701. https://doi.org/10.1021/jf049504x

[32] Moran, R.E. and Mcmanus, P. (2005) Firmness Retention and Prevention of Core Line Browning and Senescence in Macoune Apples with 1-Methylcyclopropen. Horticultural Science, 40, 161-163.

[33] Moran, R.E. and McManus, P. (2005) Firmness Retention and Prevention of Core Line Browning and Senescence, "Macoun" Apples with 1-Methylcyclopropene on Ripening of Greenhouse Tomatoes at Three Storage Temperatures. Post Harvest Biology and Technology, 27, 285-292.

[34] Zand, A.F. and Hajnajari, H. (2011) Investigation on Morphological and Pomological Traits of Promising Apple Genotypes in Karaj.

[35] Shaykh-al-Eslami, Z. (2004) Effect of Harvest Time and Storage on the Qualitative and Quantitative Properties of Apricot, CV Shahroudi. Journal of Technique Engineering Research, 6, 31-44.

[36] Akbudak, B., Ozer, M.H., Erturk, U. and Cavusoglu, S. (2009) Response of 1-Methylcyclopropene Treated "Granny Smith" Apple Fruit to Air and Controlled Atmosphere Storage Conditions. Journal of Food Quality, 32, 18-33. https://doi.org/10.1111/j.1745-4557.2008.00233.x

[37] Kondo, S., Setha, S., Rudell, D.R., Buchanan, D.A. and Mattheis, J.P. (2005) Aroma Volatile Biosynthesis in Apples Affected by 1-MCP and Methyl Jasmonate. Post harvest Biology and Technology, 36, 61-68. https://doi.org/10.1016/j.postharvbio.2004.11.005

[38] Mattheis, J.P., Xuetong, F. and Argenta, L.C. (2005) Interactive Responses of Gala Apple Fruit Volatile Production to Controlled Atmosphere Storage and Chemical Inhibition of Ethylene Action. Journal of Agricultural and Food Chemistry, 53, 4510-4516. https://doi.org/10.1021/jf0501210 
Submit or recommend next manuscript to SCIRP and we will provide best service for you:

Accepting pre-submission inquiries through Email, Facebook, LinkedIn, Twitter, etc. A wide selection of journals (inclusive of 9 subjects, more than 200 journals)

Providing 24-hour high-quality service

User-friendly online submission system

Fair and swift peer-review system

Efficient typesetting and proofreading procedure

Display of the result of downloads and visits, as well as the number of cited articles Maximum dissemination of your research work

Submit your manuscript at: http://papersubmission.scirp.org/

Orcontact as@scirp.org 